\title{
Wide range frequency tuning in vacuum electronic devices
}

V.G.Baryshevsky, K.G.Batrakov, N.A.Belous, A.A.Gurinovich, A.S.Lobko, P.V.Molchanov, P.F.Sofronov, V.I.Stolyarsky

Research Institute for Nuclear Problems, Belarussian State University, Minsk, Belarus 


\section{Contents}

$>$ Common possibilities of frequency tuning

$>\quad$ What is Volume Free Electron Laser (VFEL)

$>$ Principles of frequency tuning in VFEL

$>$ Experimental results

$>$ Future trends 


\section{Common possibilities of frequency tuning}

Operating principles of RF vacuum electronic devices suppose exploitation of distributed interactions between streaming electrons and co-traveling electromagnetic waves in resonant cavities and waveguides.

Conventional vacuum electronic systems, either amplifiers or generators, provide tuning of radiation frequency mainly due to the change of electron energy and have slight possibility of frequency tuning at fixed energy of electron beam, and this is strong intrinsic restriction of devices with one-dimensional distributed feedback. 


\section{What is Volume Free Electron Laser?}

The distinction of volume free electron laser (VFEL) is the use of one-, two- and three-dimensional diffraction gratings as volume resonators which provide volume (non-one-dimensional) multi-wave distributed feedback and, as a result, possibility of smooth frequency tuning and utilization of electron beams with large crosssection.

All known mechanisms of spontaneous radiation can be used for VFEL lasing, for example:

nonrelativistic electron beam +

+ undulator +

+ volume distributed feedback = $=$ volume ubitron

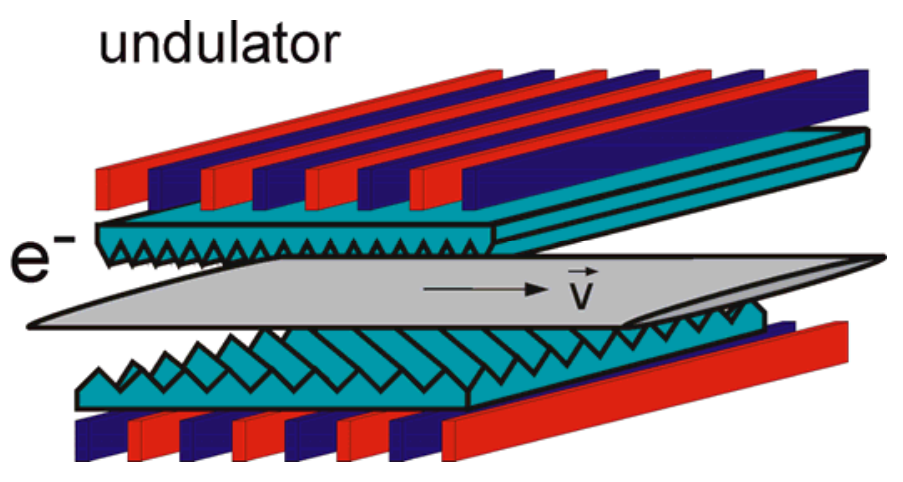
and similar for all other FEL types, for example, volume gyrotrons 


\section{What is volume distributed feedback ?}

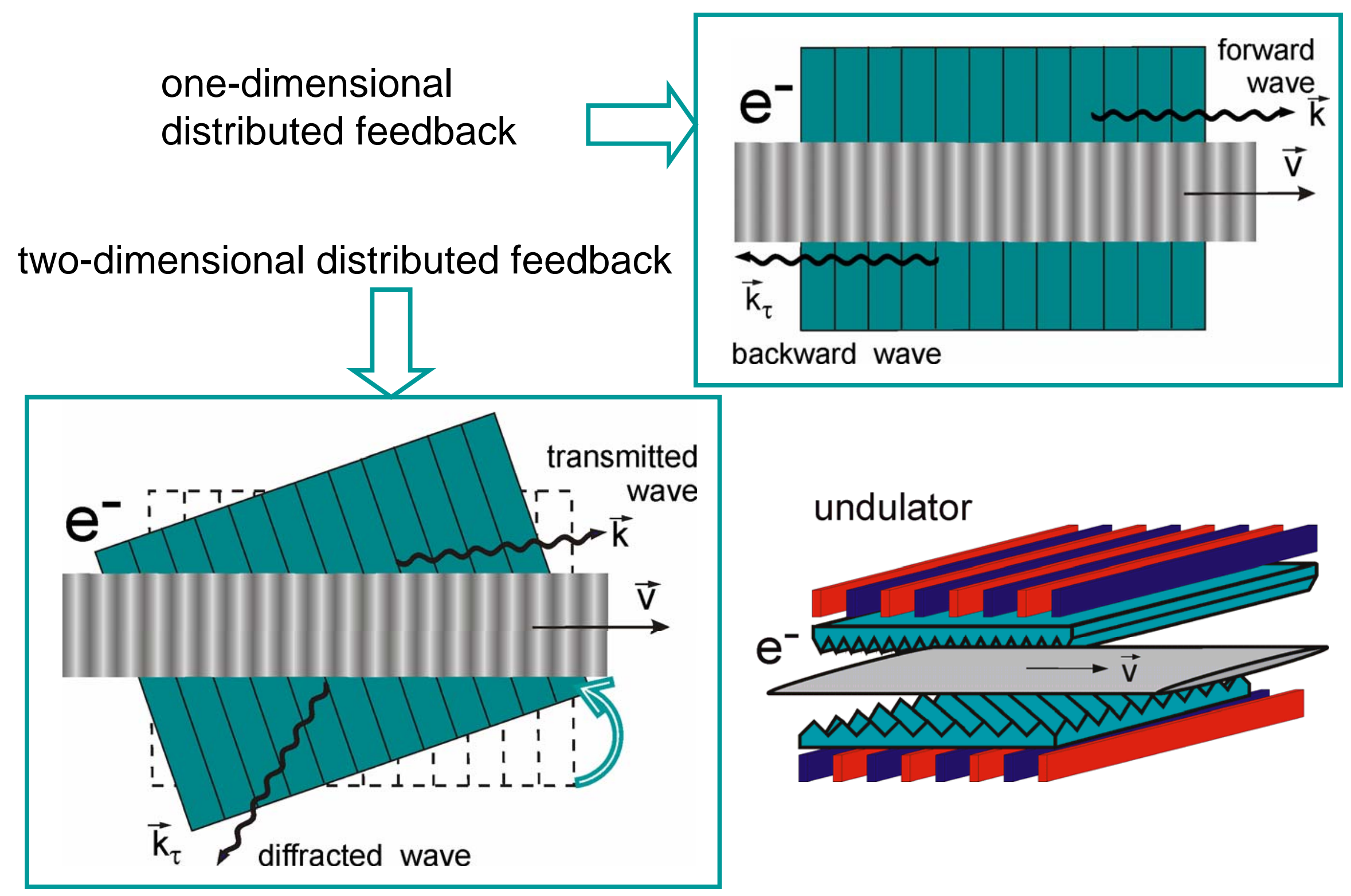




\section{Use of volume distributed feedback makes available some new features:}

- frequency tuning at fixed energy of

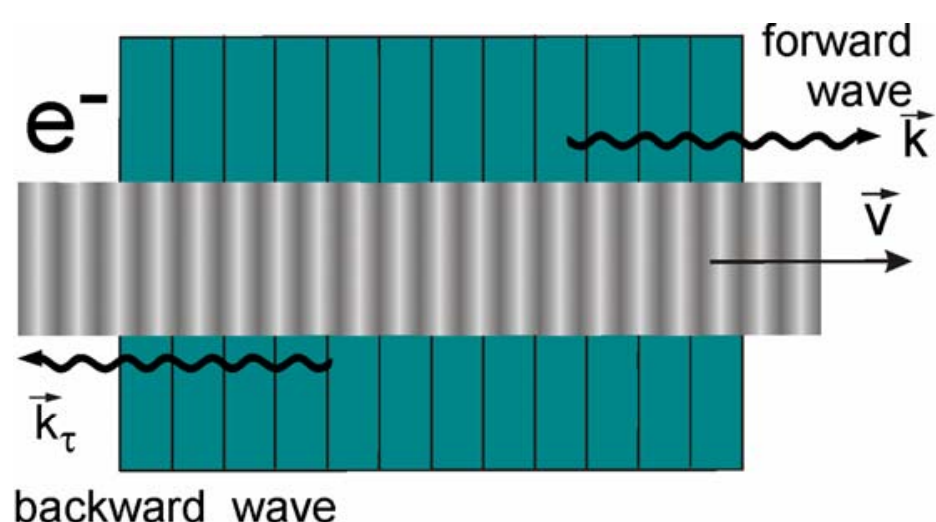

backward wave

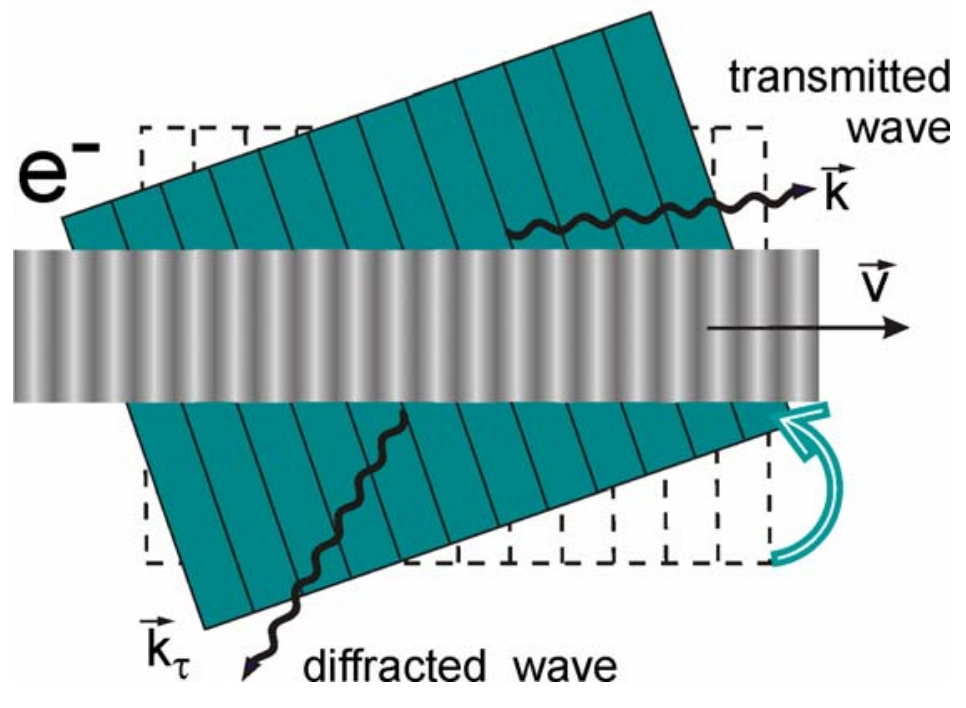
electron beam in significantly wider range than conventional systems can provide

- more effective interaction of electron beam and electromagnetic wave that allows significant reduction of threshold current of electron beam and, as a result, miniaturization of generator

- reduction of limits for available output power by the use of wide electron beams and diffraction gratings of large volumes

- simultaneous generation at several frequencies 


\section{First VFEL resonator}

First lasing of volume free electron laser in mm-wavelength range was observed in Research Institute of Nuclear Problems in 2001.

First VFEL resonator is formed by two parallel diffraction gratings with different periods and two smooth sidewalls. The interaction of the exciting diffraction grating with an electron beam arouses Smith-Purcell radiation, while resonant diffraction grating provides distributed feedback of generated radiation with electron beam by Bragg dynamical diffraction. Gratings can rotate to change orientation of grating grooves with respect to electron beam velocity that provides tuning of diffraction conditions.

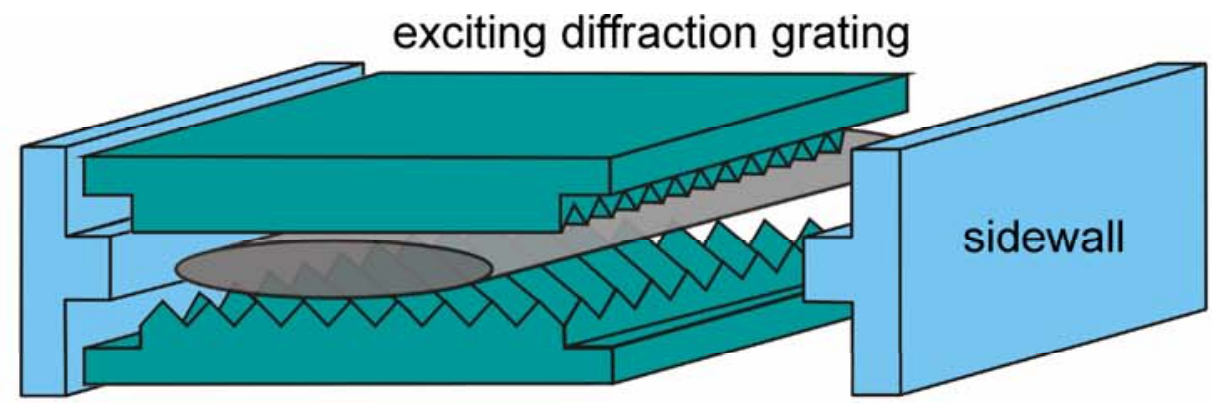

resonant diffraction grating

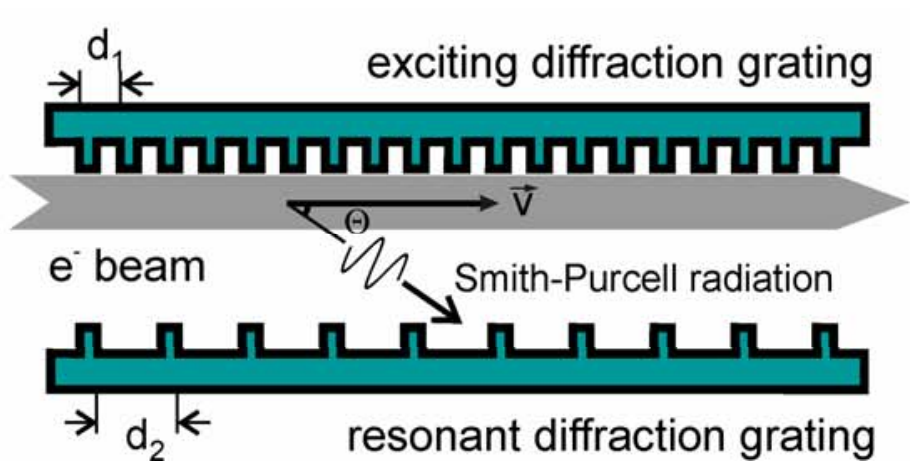




\section{Processes in resonator}

Considering processes in resonator one should discern the difference between two cases:

(a) the presence of resonator sidewalls can be neglected

(b) the presence of sidewalls of resonator can not be neglected
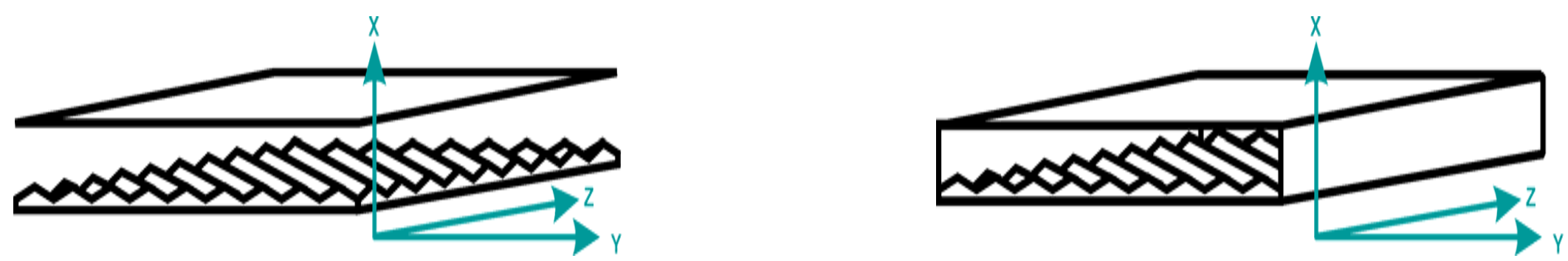


\section{(a) the presence of resonator sidewalls can be neglected}

Two geometries of diffraction are distinguished: those of Bragg and Laue

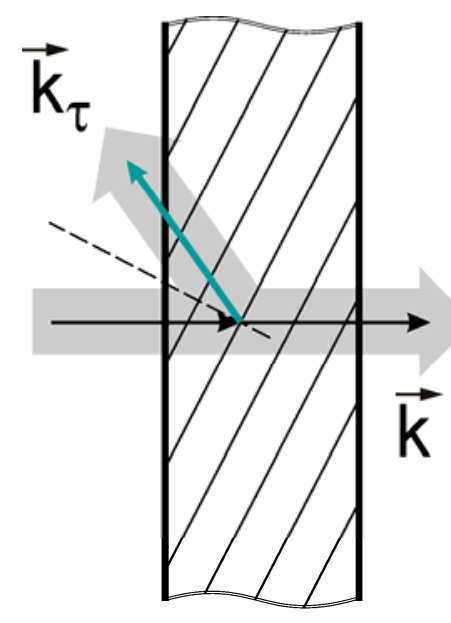

Bragg case

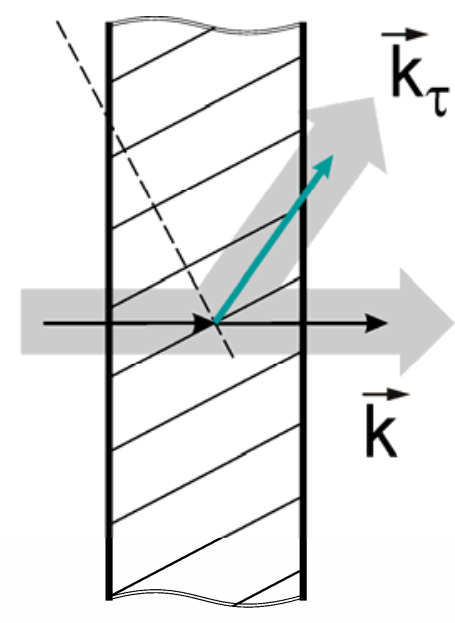

Laue case
Absolute instability can appear in Bragg geometry and such system works as a generator (for backward Bragg diffraction it converts into a well-known backward-wave tube). For Laue case convective instability provides only amplification regime. 


\section{(b) the presence of sidewalls of resonator can not be neglected}

Presence of diffraction grating in a waveguide makes possible transitions between eigenmodes of bounded resonator.

Presence of sidewalls imparts new feature to the diffraction process in resonator, because generation regime can be reached even at Laue-like geometry, in contrast to an unbounded waveguide, where Laue geometry provides only amplification regime, while generation is possible at Bragg diffraction. 


\section{Frequency tuning in present VFEL}

Frequency tuning is realized by rotation of resonant diffraction grating in the plane (YOZ). Interaction of the electron beam with the exciting diffraction grating arouses Smith-Purcell radiation

$$
\lambda=d_{1}(1 / \beta-\cos \theta),
$$

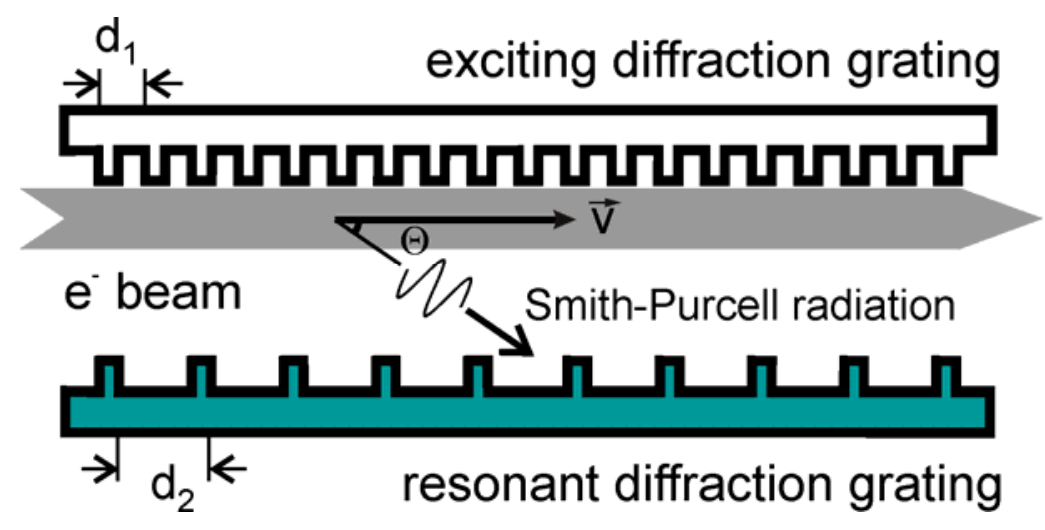

$d_{1}$ is the period of exciting diffraction grating and $\beta=V / c$.

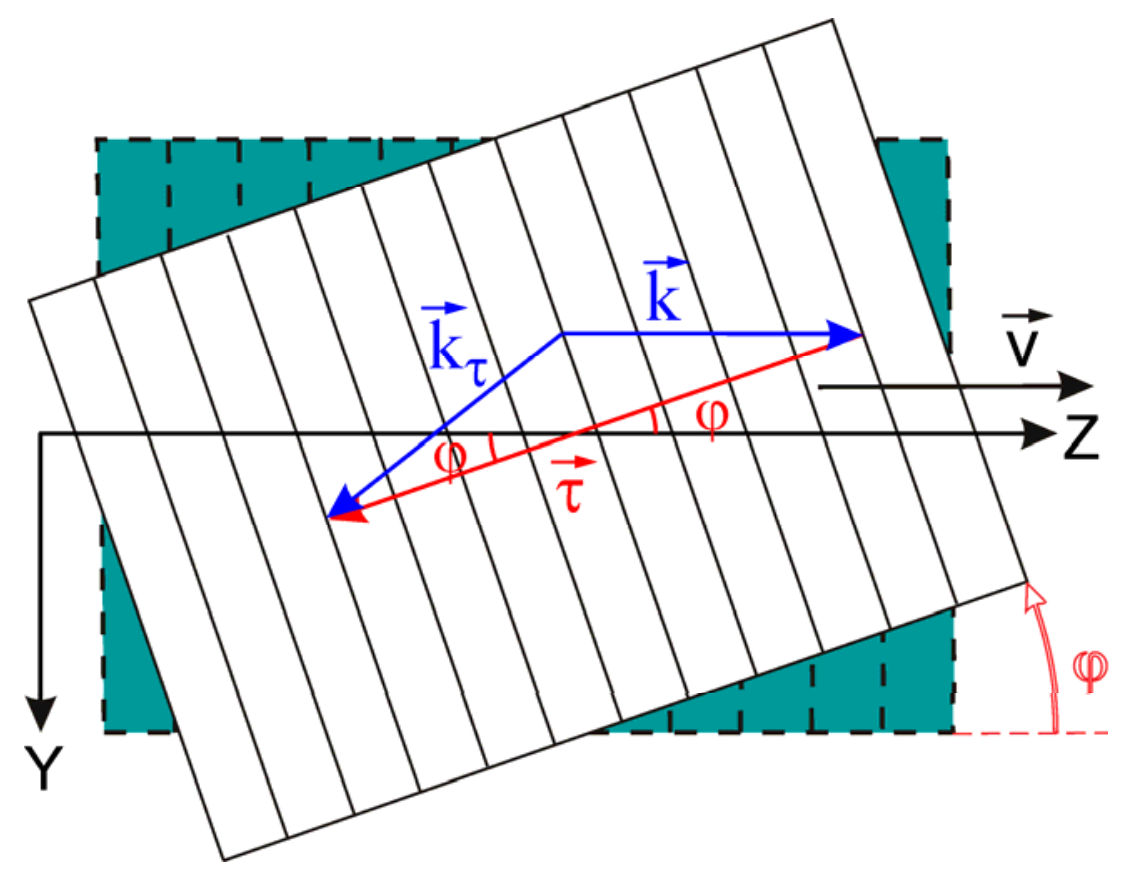

Bragg diffraction condition

$$
|\vec{k}+\vec{\tau}|=|\vec{k}|,\left(k_{z}+\tau_{z}\right) / k_{z}<0
$$

"chooses" proper $k_{z}$ for each orientation of grating grooves with respect to electron beam velocity (i.e. each reciprocal lattice vector $\vec{\tau}$ and the radiation wavelength $\lambda$ turns out $a$ function of the angle of grating rotation $\varphi$. 


\section{Frequency tuning in VFEL}

One more parameter allowing to change

$\left.\rightarrow\right|_{1} ^{d} k$

exciting diffraction grating radiation wavelength (frequency) is available in the above VFEL: $\lambda=d_{1}\left(\varphi_{1}\right)(1 / \beta-\cos \theta)$, where $d_{1}\left(\varphi_{1}\right)=d_{1} / \cos \varphi_{1}$. Tuning range in this case

$$
\Delta \lambda=\lambda\left(\varphi_{1}\right)-\lambda\left(\varphi_{1}=0\right)=\left(\frac{1-\cos \varphi_{1}}{\cos \varphi_{1}}\right) d_{1}\left(\frac{1}{\beta}-\cos \theta\right)
$$

exciting grating
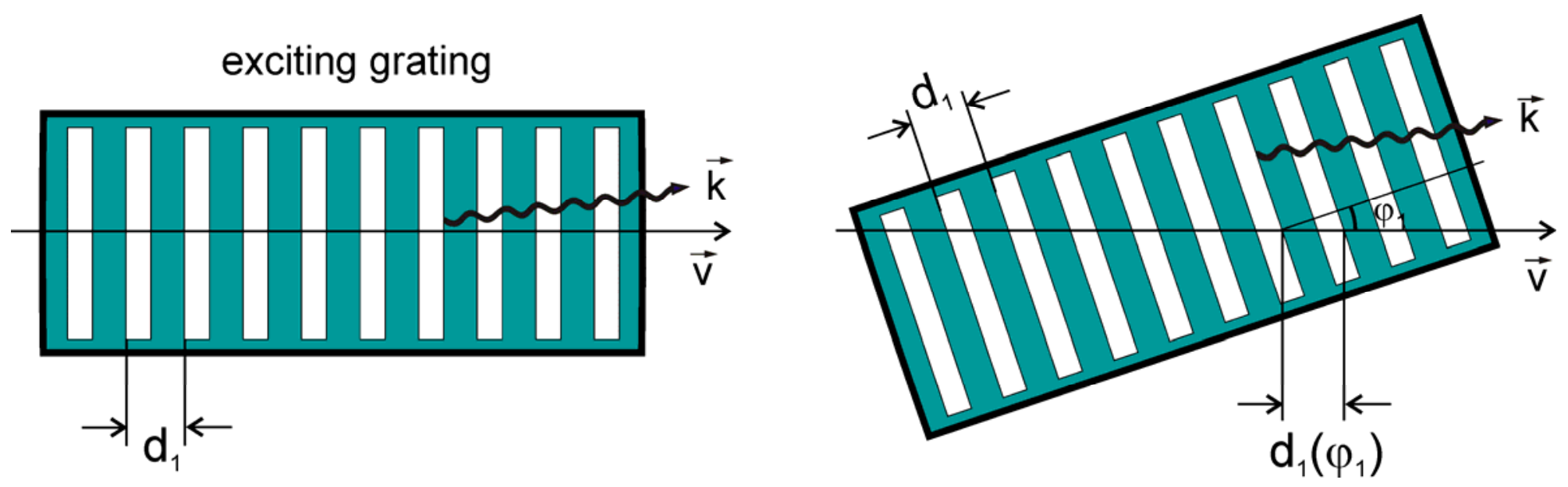


\section{Experimental results}

Measured frequencies at rotation of resonant diffraction grating for microwave peak, corresponding electron beam energy 2,9 keV.

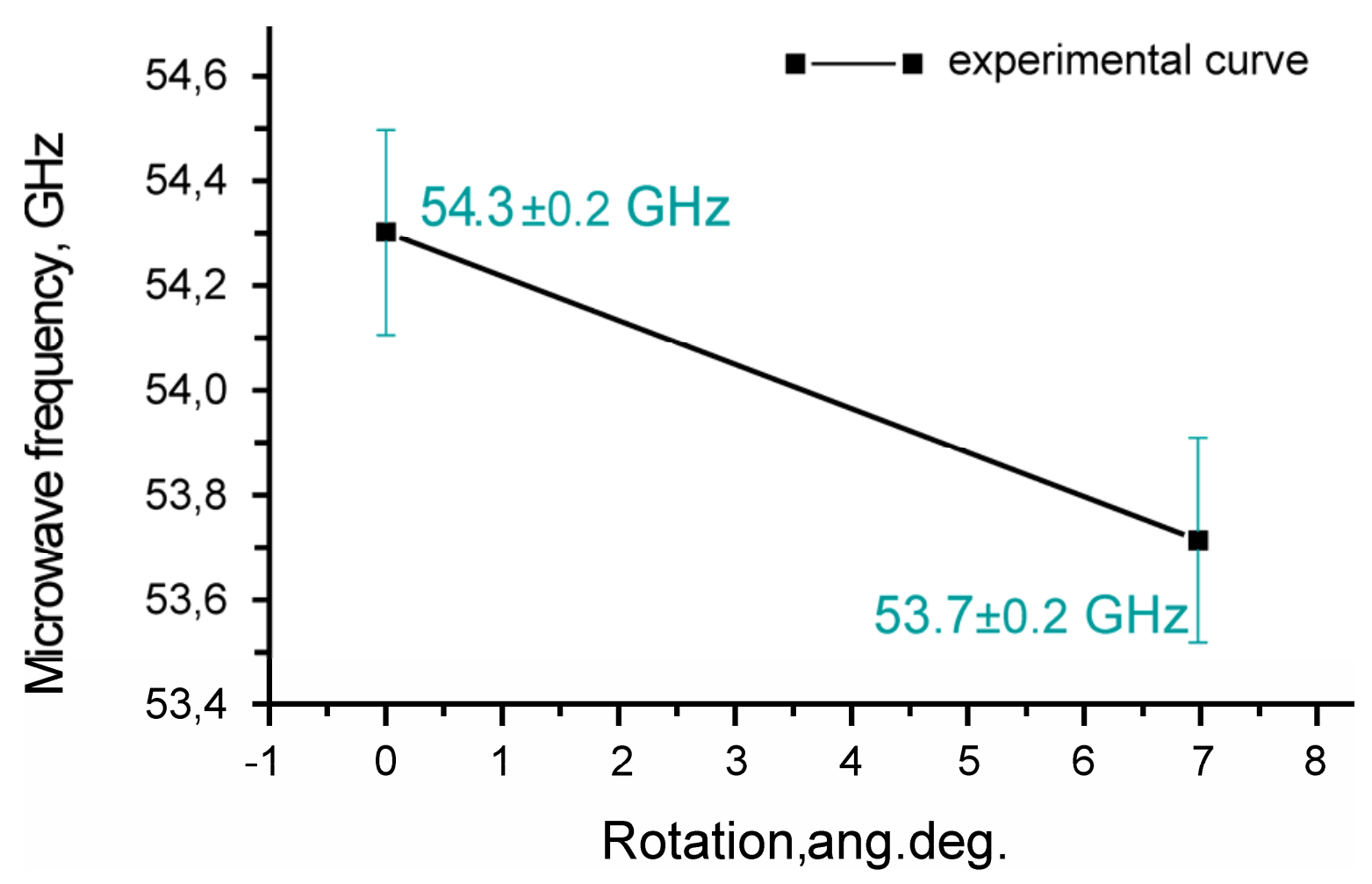

Measured frequency change well accords theoretical predictions 


\section{Experimental results}

Radiation frequencies for different electron beam energies

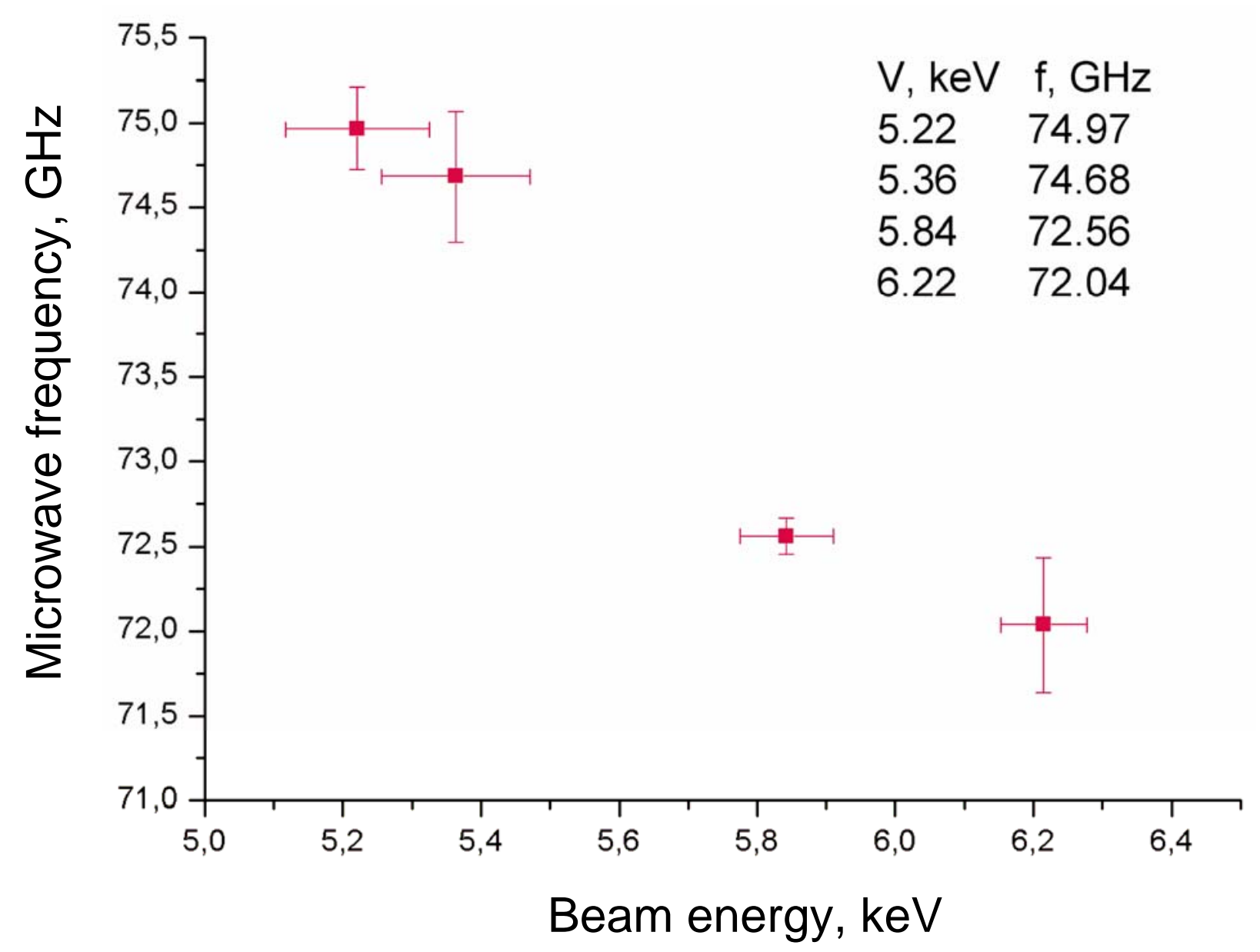




\section{Experimental results}

Radiation frequency change at rotation of resonant diffraction grating

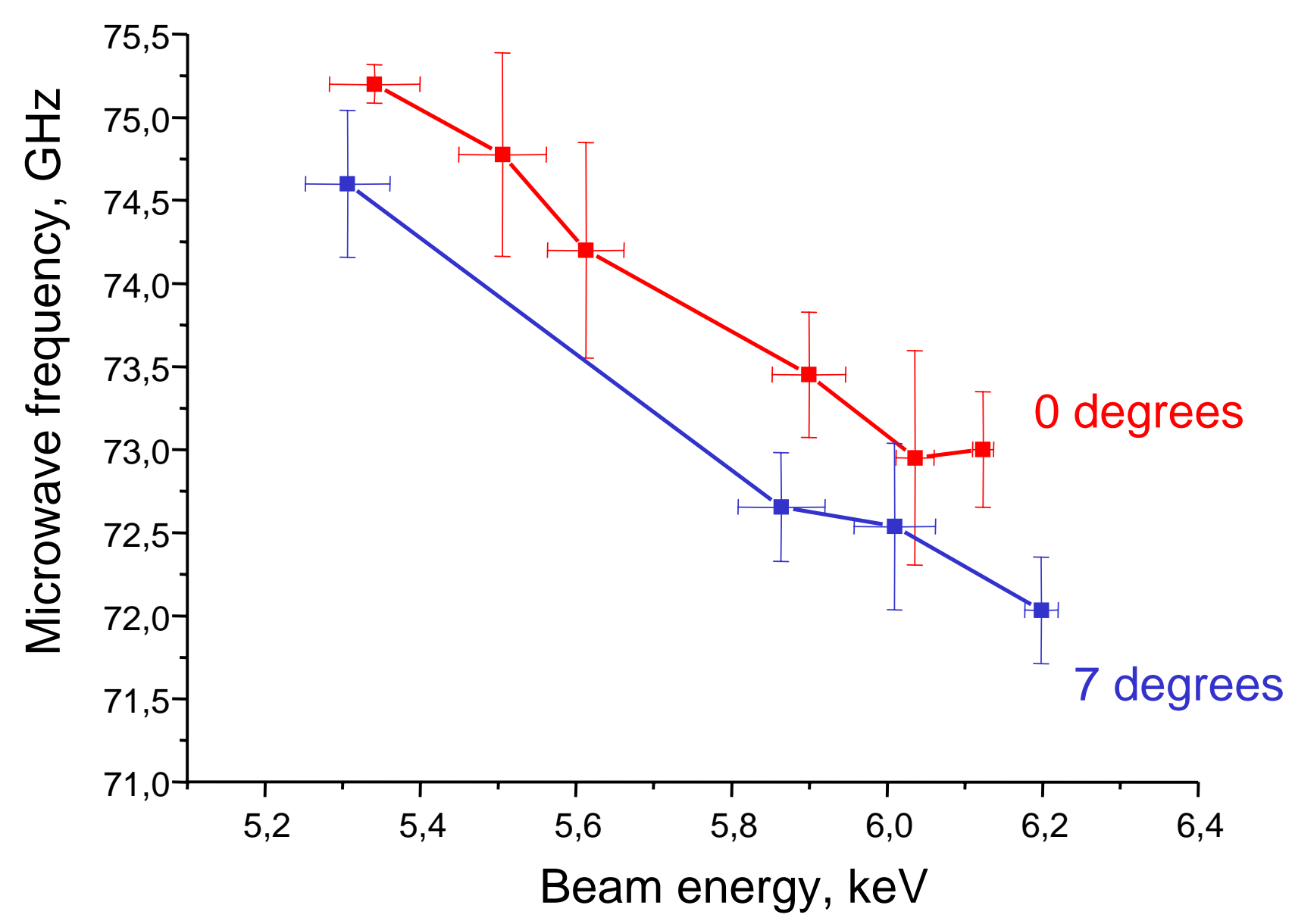




\section{VFEL types}
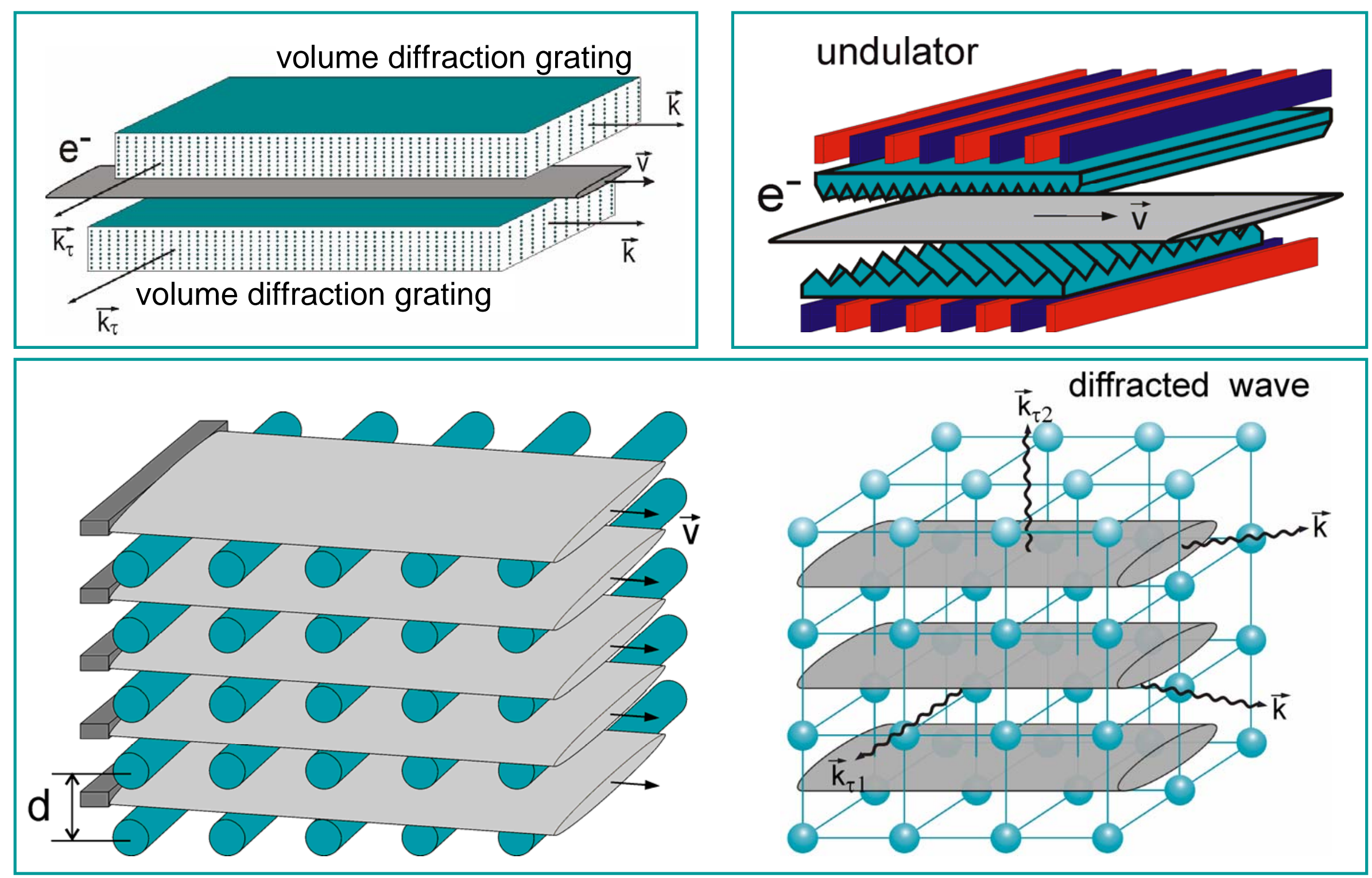

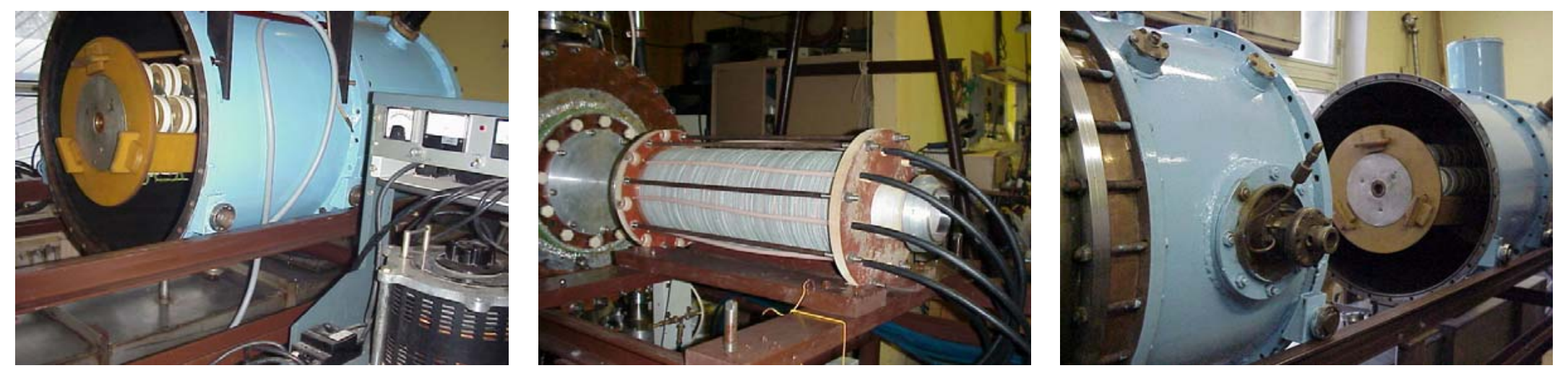

\section{New VFEL generator}

with electron beam energy $300 \mathrm{keV}$ is ready to start
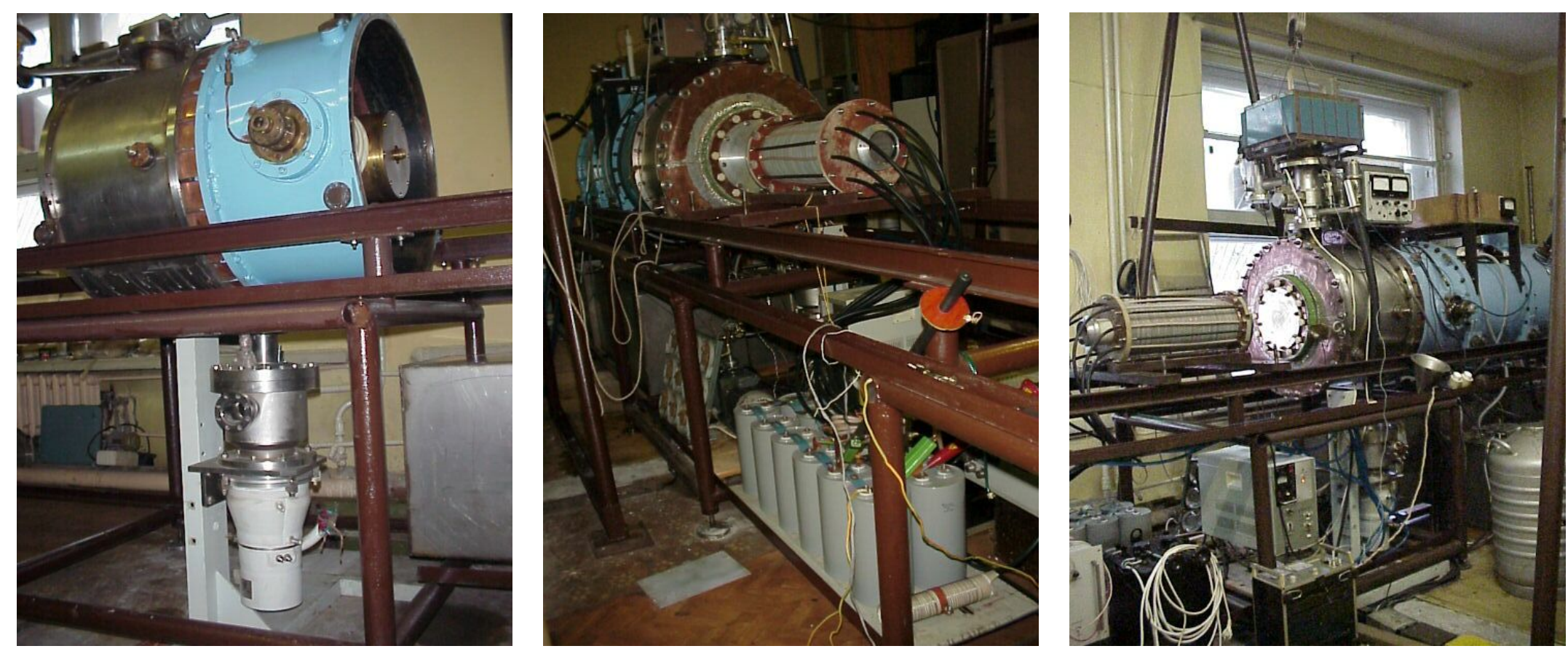


\section{Conclusion}

Volume Free Electron laser is a new type of FEL using volume distributed feedback instead of one-dimensional that.

Possibility of wide range frequency tuning (due to gratings rotation), provided by volume distributed feedback allows us to propose VFEL generator as a new type of tunable backward wave tube (ubitron, gyrotron) and VFEL amplifier as a new type of tunable travelling wave tube (ubitron, gyrotron).

VFEL merits make it a basis for development of more compact, highpower and tunable radiation sources then conventional electron vacuum devices could let. 